\title{
Comparative Secretome Profiling and Mutant Protein Identification in Metastatic Prostate Cancer Cells by Quantitative Mass Spectrometry-based Proteomics
}

\author{
OH KWANG KWON, JU MI JEON, EUNJI SUNG, ANN-YEA NA, SUN JOO KIM and SANGKYU LEE \\ College of Pharmacy, Research Institute of Pharmaceutical Sciences, \\ BK21 Plus KNU Multi-Omics-based Creative Drug Research Team, \\ Kyungpook National University, Daegu, Republic of Korea
}

\begin{abstract}
Background: Secreted proteins play an important role in promoting cancer $(\mathrm{PCa})$ cell migration and invasion. Proteogenomics helps elucidate the mechanism of diseases, discover therapeutic targets, and generate biomarkers for diagnosis through protein variations. Materials and Methods: We carried out a mass spectrometry-based proteomic analysis of the conditioned media $(C M)$ from two human prostate cancer cell lines, belonging to different metastatic sites, to identify potential metastatic and/or aggressive factors. Results: We identified a total of 598 proteins, among which 561 were quantified based on proteomic analysis. Among the quantified proteins, 128 were up-regulated and 83 were down-regulated in DU145/PC3 cells. Six mutant peptides were identified in the CM of prostate cancer cell lines using proteogenomics approach. Conclusion: This is the first proteogenomics study in PCa aiming at exploring a new type of metastatic factor, which are mutant peptides, predicting a novel biomarker of metastatic PCa for diagnosis, prognosis and drug targeting.
\end{abstract}

Prostate cancer (PCa) is the second most frequent cancer in men worldwide, with lung cancer topping the list. The American Cancer Society has reported that PCa accounted for approximately 180,890 new cases in 2016, with an estimated 26,120 deaths only in the United States (1).

This article is freely accessible online.

Correspondence to: Sangkyu Lee, Ph.D., Associate Professor, College of Pharmacy, Research Institute of Pharmaceutical Sciences, Kyungpook National University, 80 Daehak-ro, Buk-gu, Daegu 41566, Republic of Korea. Tel: +82 53950 8571, Fax: +82 539508557, e-mail: sangkyu@knu.ac.kr

Key Words: Prostate cancer, metastasis, MS-based proteomics, proteogeomics, DU145.
Twenty-five percent of patients progress from initial hormone sensitivity to castrate-resistant prostate cancer (CRPC), that no longer accepts hormone therapy (2). Consequently, metastatic castration-resistant prostate cancer (mCRPC) is considered incurable; many of these patients develop recurrence with a poor outlook, eventually leading to death. Metastasis of cancer is a multi-step cascade that includes local migration, invasion, and colonization. $\mathrm{PCa}$ is able to metastasize to liver, lung, and brain, but almost proprietarily associates with a high incidence of bone metastases (3). Despite many studies on mCRPC, the metastatic mechanism and/or involved factors are still not clear. Hence, metastatic studies and identification of diagnostic factors in $\mathrm{PCa}$ are of increasingly high priority.

Secretory proteins play an important role in cancer metastasis by stimulating cancer cell migration and invasion capability, consequently increasing the cancer metastasis in the extracellular microenvironment (4). Several researchers have reported the relation of secretory proteins with cancer metastasis, suggesting their application as putative tumor biomarkers and/or in understanding pathophysiological pathways (5-9). A big challenge in the diagnosis of $\mathrm{PCa}$ is the lack of alternative screening to replace the existing $\mathrm{PCa}$ biomarker prostate-specific antigen (PSA). Although widely used, PSA cannot distinguish between indolent and aggressive PCa (10). For this reason, exploring biomarkers, especially metastasis-associated factors, is imperative for prostate cancer research.

Cancer- or onco-proteogenomics combines mass spectrometry-based proteomics with genomic information to study protein variations and cancer-specific mutant proteins. Proteogenomics can help researchers understand the underlying mechanisms of diseases, discover therapeutic targets, or generate biomarkers for diagnosis or tailored therapies $(11,12)$. Generally, identification of mutant proteins by mass spectrometry-based proteomics has been utilized by de novo sequencing or database search method 
with genomics and trascriptomics. De novo sequencing algorithms can directly indicate amino acid sequence without database matching, but its accuracy is not reliable enough, mostly due to ambiguous interpretations of MS/MS spectra (13). However, database search algorithms, associated with cancer-related mutation database, such as Unknown peptidelevel Mutation Analysis (XMAn), Human Cancer Proteome Variation Database (CanProVar), and Cancer Mutation Proteome Database (CMPD), can be used to identify the relevant mutant proteins (14-16).

In this study, we adopted a proteomics approach for the conditioned media from two human prostate cancer cell lines of differing metastatic origins [DU145 (brain metastasis) and PC3 (bone metastasis)] to identify the putative metastatic factors, including secreted and/or mutant proteins, using quantitative mass spectrometry. We identified a total of 598 secretory proteins, out of which 561 were quantified based on proteomic analysis. Among the quantified proteins, 128 were up-regulated and 83 were down-regulated in DU145/PC3. Of these, laminin gamma 1 (LAMC1) was significantly elevated in DU145 cell media. Also, MS/MS spectra were re-analyzed by MaxQuant with CMPD to find out prostate cancer-specific mutant proteins in different cell lines. As a consequence, we discovered six mutant peptides, two of which are assumed to be associated with metastasis and/or aggressiveness in DU145 cells. We propose that LAMC1 and the six mutant peptides are not only able to distinguish metastatic sites, but are also likely to be used for drug targeting in prostate cancer.

\section{Materials and Methods}

Cell culture. The human prostate cancer cell lines, DU145 and PC3, were purchased from the Korean Cell Line Bank (KCLB, Seoul, Korea). Cells were cultured in RPMI-1640 (GE healthcare, Little Chalfont, UK) supplemented with $10 \%(\mathrm{v} / \mathrm{v})$ fetal bovine serum (Gibco, Rockville, MD, USA) and $1 \%$ penicillin-streptomycin (Gibco) at $37^{\circ} \mathrm{C}$ with $5 \% \mathrm{CO}_{2}$ in a humidified incubator.

Enrichment of secretory proteins from conditioned media. DU145 and PC3 cells were grown to $\sim 70 \%$ confluence $\left(\sim 3.5 \times 10^{6}\right.$ cells $)$ in $150 \mathrm{~mm}$ culture dishes (SPL Life Science, Pocheon, Republic of Korea). The cell monolayer was rinsed carefully with Dulbecco's Phosphate-Buffered Saline (Gibco) and serum-free medium (SFM) to avoid serum contamination. The cells were incubated in SFM at $37^{\circ} \mathrm{C}$ for $24 \mathrm{~h}$. After incubation, the conditioned media from three plates were carefully collected and protease inhibitors (Thermo Fisher Scientific, Boston, MA, USA) added. Floating cells and cellular debris were removed by centrifugation at $3,000 \times g$ for $10 \mathrm{~min}$ at $4^{\circ} \mathrm{C}$. The supernatants were concentrated by ultrafiltration using 10K MWCO protein concentrators (Thermo Fisher Scientific). The protein concentrations of conditioned media samples from DU145 and PC3 were determined by the BCA protein assay (Thermo Fisher Scientific). All protein samples were stored at $-80^{\circ} \mathrm{C}$ until further use.
Western blotting. Protein samples, containing $5 \mu \mathrm{g}$ of cell lysate and secretory proteins from conditioned media, were separated by $10 \%$ SDS-PAGE and transferred to PVDF membranes (Roche, Basel, Switzerland). All membranes were blocked by $5 \%$ BSA, TBST buffer (20 mM Tris, $147 \mathrm{mM}$ sodium chloride, $0.1 \%$ Tween-20) for $3 \mathrm{~h}$ at RT and incubated overnight at $4^{\circ} \mathrm{C}$ with primary antibodies. All antibodies were diluted to 1:1,000 with 5\% BSA in TBST. The membranes were washed thrice in TBST for $15 \mathrm{~min}$ each, incubated with corresponding IgG-HRP secondary antibodies at a dilution of 1: 2,000 for $1 \mathrm{~h}$ at RT, washed, and finally visualized with the ECL plus as chemiluminescent substrate (GE Healthcare, Uppsala, Sweden). The primary antibodies used include anti- $\beta$-actin from Cell Signaling Technology (Danvers, MA), anti- $\alpha$-tubulin from Abcam (Cambridge, UK), anti-Serpin B3 from Thermo Fisher Scientific and anti-LamC1 from OriGene (Rockville, MD, USDA). The secondary antibodies used were anti-mouse IgG-HRP (Cell Signaling Technology, Danvers, MA, USA) and anti-rabbit IgGHRP (Cell Signaling Technology).

Sample preparation for quantitative proteomics. The protein samples $(25 \mu \mathrm{g})$ were reduced with $200 \mathrm{mM}$ Tris(2-carboxyethyl)phosphine (TCEP) at $55^{\circ} \mathrm{C}$ for $1 \mathrm{~h}$ and alkylated with $60 \mathrm{mM}$ iodoacetamide (IAA) for $30 \mathrm{~min}$, protected from light at room temperature. Afterwards, the proteins were precipitated with six volumes of prechilled acetone for $4 \mathrm{~h}$ at $-20^{\circ} \mathrm{C}$. After centrifugation, the pellet obtained was dissolved in $100 \mathrm{mM}$ TEAB. The protein was digested by sequencing-grade modified trypsin (Promega, Madison, WI, USA) at $37^{\circ} \mathrm{C}$ for $16 \mathrm{~h}$. The ratio of enzyme to protein was $1: 50$.

The digested peptides were labeled with a six-plex TMT isobaric Mass Tagging Kit (Thermo Fisher Scientific) according to the manufacturer's protocol. Briefly, $41 \mu \mathrm{l}$ of anhydrous acetonitrile was added to each tube. Reagents were dissolved by vortexing for $5 \mathrm{~min}$. Protein samples were labeled by adding $20 \mu$ of TMT Label Reagent (PC3: 127, DU145: 129) and incubated for $1 \mathrm{~h}$ at room temperature. To quench the reaction, 5\% hydroxylamine was added, followed by 15 -min incubation at RT. The TMT labeled peptide mixtures were dried by vacuum centrifugation. The peptide was fractionated by High pH Reversed-Phase Peptide Fractionation Kit (Thermo Fisher Scientific) according to the manufacturer's protocol. A total of 18 fractions were cleaned with C18 ZipTips (Merck Millipore, Burlington, MA) in accordance with the manufacturer's instructions, followed by LC/MS analysis.

Nano LC-MS/MS analysis. Enriched peptides were dissolved in solvent A $(0.1 \%$ formic acid and $2 \%$ acetonitrile in water). Peptide separation was performed using a handmade $\mathrm{C} 18$ reversed-phase analytical column (Reprosil C18 $3 \mu \mathrm{m}$ beads, Dr. Maisch GmbH, Germany) with a linear gradient of $2-23 \%$ solvent B $(0.1 \%$ formic acid and $10 \%$ water in acetonitrile) for $44 \mathrm{~min}$ and $23-90 \%$ solvent B for $5 \mathrm{~min}$ at a constant flow rate of $200 \mathrm{~nL} / \mathrm{min}$ using an EASY-nLC 1000 UPLC system (Thermo Fisher Scientific). The peptides were ionized using a nanospray ion source followed by tandem MS/MS in a Q-Exactive Plus Hybrid Quadrupole-Orbitrap Mass Spectrometer (Thermo Fisher Scientific) coupled online to the UPLC. Intact peptides were detected in the Orbitrap at a resolution of 70,000. Peptides were selected for MS/MS using 27\% normalized higher-energy collisional dissociation (HCD); ion fragments were detected in the Orbitrap at a resolution of 17,500. A data-dependent procedure that alternated between one MS scan followed by $15 \mathrm{MS} / \mathrm{MS}$ scans was applied for the top 15 precursor ions above a threshold ion count of 3E6 in the MS survey scan with a 
$25 \mathrm{~s}$ dynamic exclusion. The electrospray voltage applied was $2.2 \mathrm{kV}$ Automatic gain control was used to prevent overfilling of the ion trap; 1E5 ions accumulated for the generation of MS/MS spectra. For MS scans, the $\mathrm{m} / \mathrm{z}$ scan range was 300 to $1,400 \mathrm{Da}$.

MS/MS data analysis. The resulting MS/MS data were processed using MaxQuant with the integrated Andromeda search engine (v.1.5.1.0) (17). MS/MS spectra were searched against the Uniprot Human database from Uniprot (http://Uniprot.org) (18). To identity sitespecific mutant peptides, only prostate cancer-related database at NCI60 and TCGA were downloaded at CMPD (http://cgbc.cgu.edu.tw/ $\mathrm{cmpd} /$ ) (16) and applied in MaxQuant. For MaxQuant searching, Trypsin/P was specified as the cleavage enzyme and up to two missed cleavages, five modifications per peptide, and seven charges were allowed. Mass error was set at $10 \mathrm{ppm}$ for precursor ions and $0.02 \mathrm{Da}$ for fragment ions. Carbamidomethylation on Cys was specified as a fixed modification, whereas oxidation on Met and acetylation on protein $\mathrm{N}$-termini were specified as variable modifications. Falsediscovery rate thresholds for protein, peptide, and modification sites were specified at $1 \%$. Minimum peptide length was set at 7 . For quantification of peptides by TMT, the reporter ions were calculated by six-plex TMT at peptide N-termini and lysine. All the other parameters in MaxQuant were set to default values.

Bioinformatics analyses. The identified proteins were annotated by the Kyoto Encyclopedia of Genes and Genomes (KEGG), InterPro, and Gene Ontology (GO), which consist of GO molecular function (GOMF), GO biological process (GOBP), and GO cellular component (GOCC) using Perseus 1.6 (19). For functional enrichment of differentially quantified proteins, DAVID 6.7 webbased software, which provides comprehensive set of functional annotation tools for investigators to understand biological meaning, was employed. Threshold of EASE score, a modified Fisher's exact test $p$-value, was smaller than 0.05 to be deliberated as high enrichment of annotation categories (20). We used the STRING software to trace the critical assessment and integration of proteinprotein interactions from the identified secretory proteins (21). The STRING diagrams, with a corrected interaction score $<0.9$, is considered significant and masked disconnected proteins in the network. Visualization, such as scatter plot, for proteomics results were drawn using Perseus 1.6, following the protocol.

\section{Results}

Quantitative secretome profiling of metastatic prostate cancer cell lines. In this study, we performed a quantitative investigation of secretory and mutant proteins associated with prostate cancer in $\mathrm{CM}$ using a mass spectrometry-based proteomics approach with TMT labeling (Figure 1A). Secretory proteins from $\mathrm{CM}$ were enriched using $10 \mathrm{~K}$ MWCO protein concentrators, which were able to concentrate and purify proteins easily. For quality check of the proteins from CM, we performed western blotting, with $\beta$-actin and $\alpha$-tubulin antibodies serving as controls for the analysis. As expected, $\alpha$-tubulin was not detected in the CM, whereas $\beta$ actin was detected in both cell lysate and CM (Figure 1B). Therefore, we could confirm that the protein samples from $\mathrm{CM}$ were not contaminated by cell debris.
For in-solution tryptic digestion, the proteins were reduced and alkylated by TCEP and IAA, respectively, followed by precipitation in ice-cold acetone for $4 \mathrm{~h}$ at $-20^{\circ} \mathrm{C}$ to remove the excess TCEP and IAA. Trypsin was added to purified proteins and incubated overnight at $37^{\circ} \mathrm{C}$. The peptides were labeled by TMT reagents to discriminate secretory proteins between DU145 and PC3 cells. To improve the identification of secretory proteins, we carried out high-pH reverse-phase peptide fractionation, which could separate eight fractions from a mixture of TMT-labeled peptides. High-resolution and accurate-mass spectrometry (HRAM), namely QExactive Hybrid Quadrupole-Orbitrap MS, was utilized with EASY-nLC 1000 UPLC system in order to analyze the peptides. To quantify the secreted proteins from PC3 and DU145 cells, we performed MaxQuant database search of the obtained MS/MS spectra against the Uniprot Human database (including 70,630 proteins) (Figure 1C). To improve the accuracy of identification, search results were filtered, allowing a maximum false detection rate of $1 \%$ and minimum score of 40 for proteins and peptides. Also, our data were replicated to minimize random noise associated with TMT labeling and LC-MS/MS equipment. The Pearson correlation coefficient $(\mathrm{R})$ of reporter ion intensity in 127 was 0.804 and that in 129 was 0.798 (Figure 2A).

Using the Uniprot Human database, we identified a total of 598 secretory proteins with at least two peptides in the combined CM of DU145 and PC3 cells, among which 561 proteins were quantified based on comparative proteomic analysis. 350 proteins (62.4\%) remained unaltered, commonly detected in both cell lines. Among the quantified proteins, $128\left(22.8 \%\right.$ ) were highly secreted (DU145 vs. PC3, $\log _{2}$ ratio $\geq 1$ ) and 83 (14.8\%) were less-secreted (DU145 vs. PC3, $\log _{2}$ ratio $\leq-1$ ) in DU145 cells, respectively (Figure 2B).

Verification and bioinformatic analysis of secretory proteins in DU145 and PC3. We confirmed the secretory proteins (detected from MS-based proteomics results) by western blot assays. From the proteomics results, LamC1 (\#P11047, ratio: 4.013) was observed to be secreted more into the CM of DU145, compared to that of PC3, whereas Serpin B3 (\#P29508, ratio: 0.752) was detected to be slightly decreased in DU145 compared to that in PC3 (Figure 2C). Western blots of LamC1 and Serpin B3 conform to the conducted quantitative proteomics analysis.

To acquire in-depth understanding of the biological functions of differentially expressed proteins, we used the DAVID web-based software and conducted an enrichment analysis of different categories, including Gene Ontology (GO), KEGG pathway, and InterPro. The 211 differentially secreted proteins, including 128 predominant in DU145 and 83 predominant in PC3, were analyzed for functional annotation enrichment, with respect to GOBP, GOMF, GOCC, KEGG, and InterPro, enlisted according to the 
A

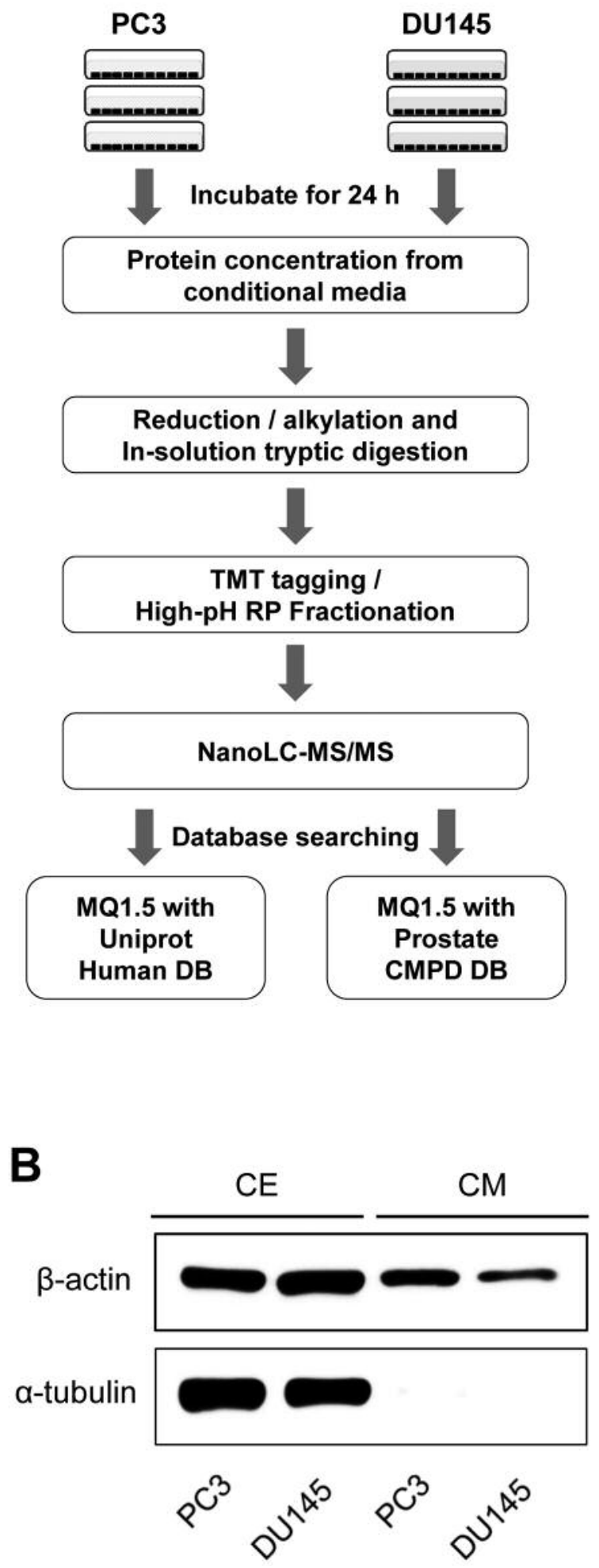

C

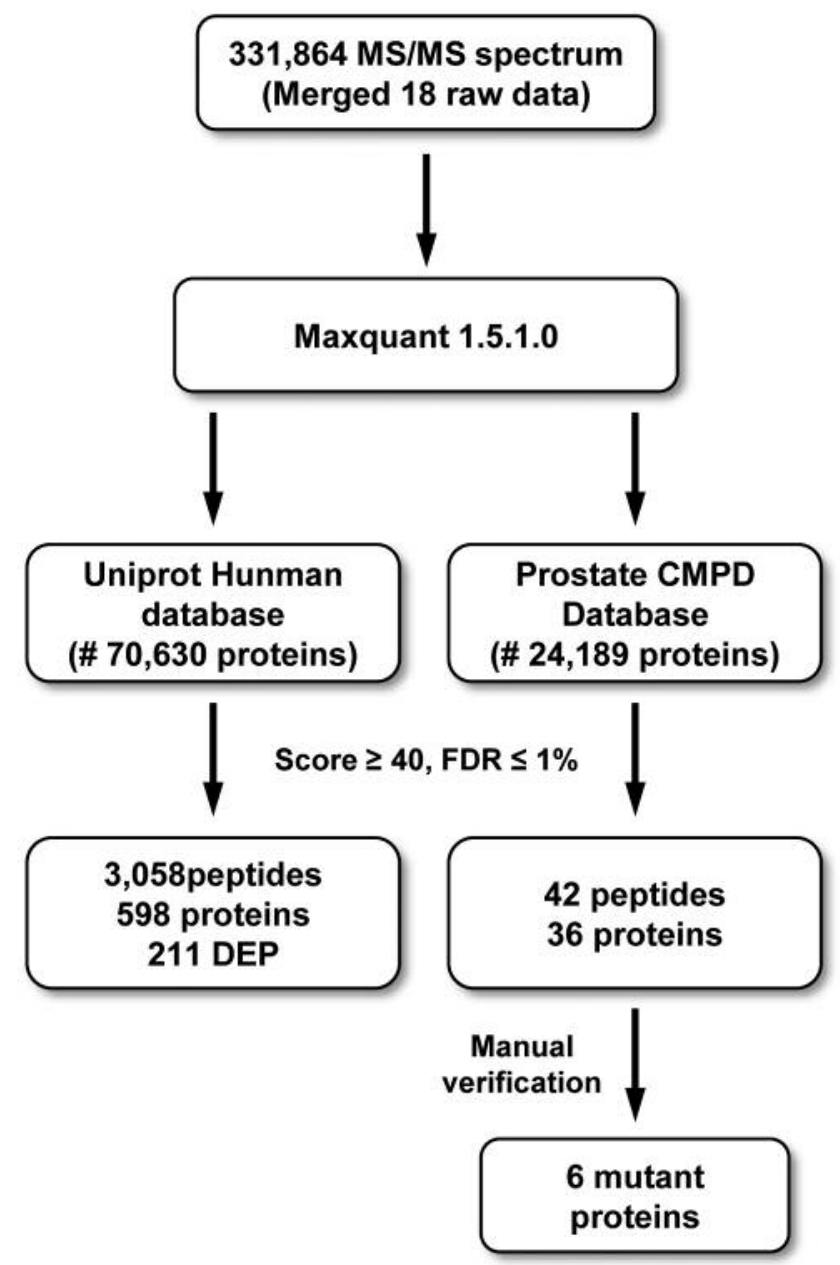

Figure 1. Comparative profiling of secreted proteins in DU145 and PC3 cells using TMT-based proteomics (A). Western blot analysis of actin and tubulin in CM and CE of PC3 and DU145 cells (B). Schematic illustration of proteomics and proteogenomics for cancer-related mutant proteins via the MaxQuant search engine $(C)$. 
A
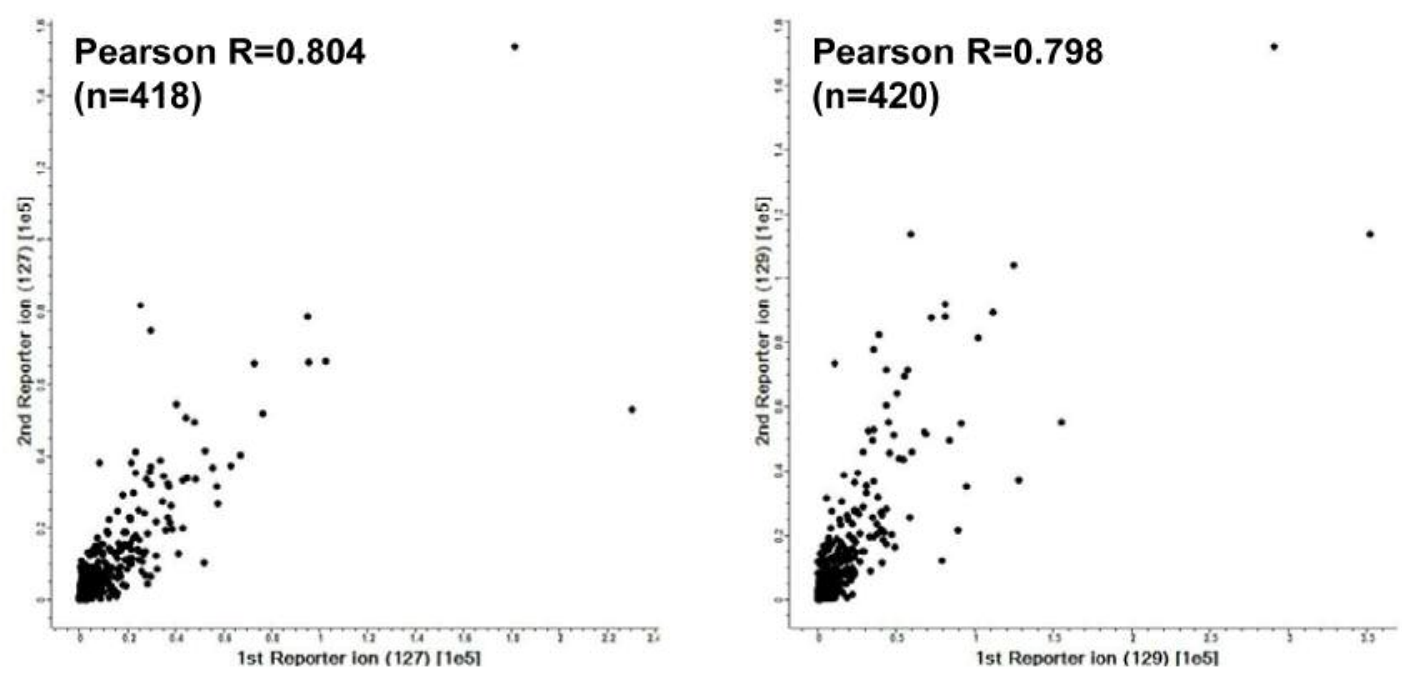

B
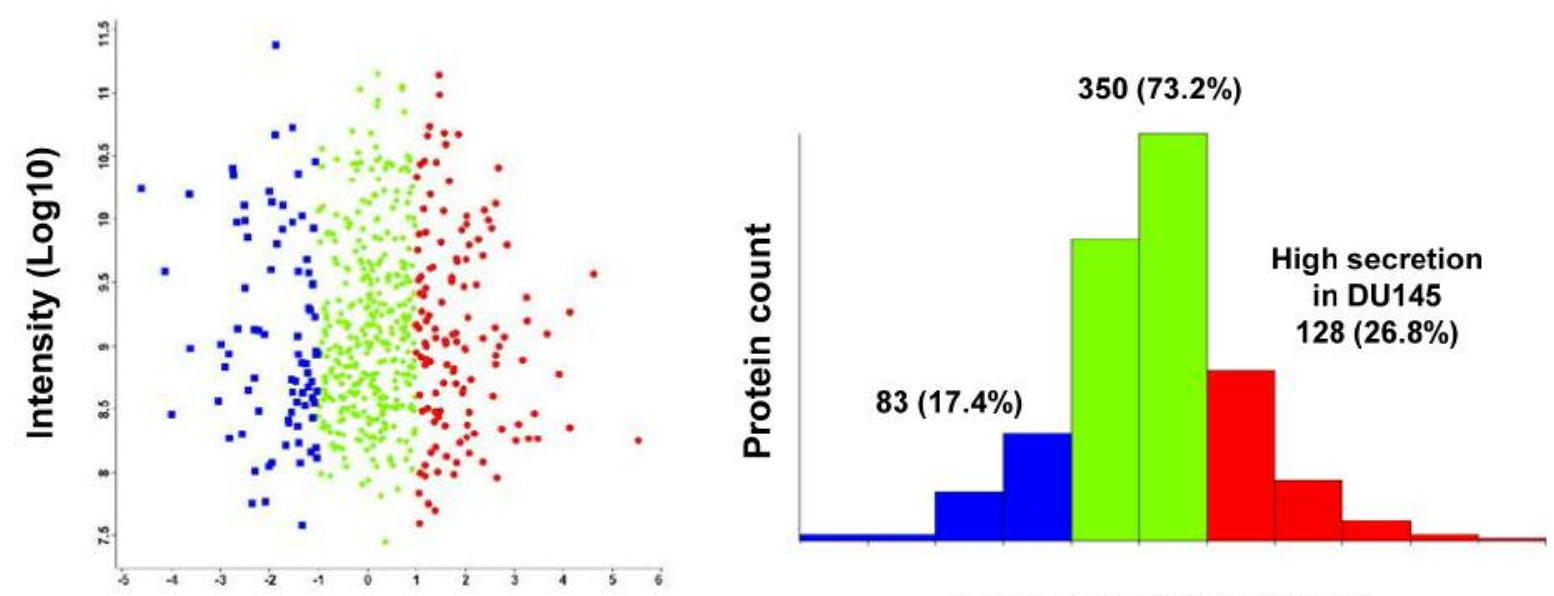

Log2 Ratio (DU145/PC3)

\section{Log2 Ratio (DU145/PC3)}

C
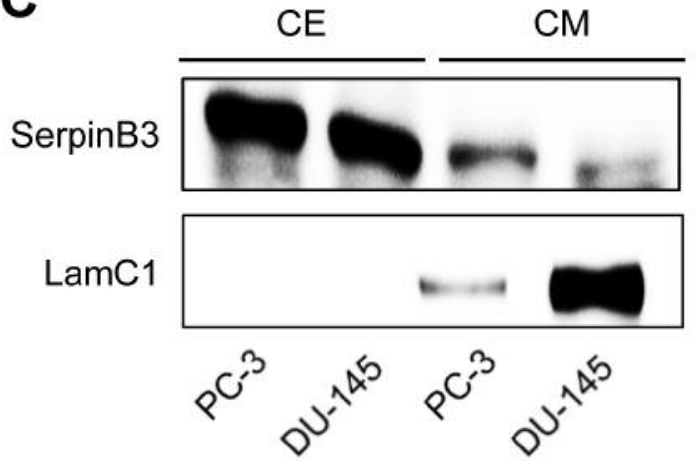

Figure 2. Pearson correlation coefficient of TMT reporter ion intensity 127 (left) and 129 (right) (A). Scatter plot and distribution of secretory proteins between PC3 and DU145 (B). Western blot analysis of SerpinB3 and LamC1 in CM and CE from PC3 and DU145 cells (C). 
differentially secreted proteins. Among these, three highest data from Fisher exact test, for each category, were extracted and summarized in Figure 3. The functional annotation enrichment of GOCC was mainly related to extracellular terms in both cell lines, as expected. Based on the enrichment results of GOMF, laminin-, integrin-, and cadherin-binding proteins were enriched in DU145 cells, whereas growth factor-related proteins were mostly secreted from PC3. The enrichment analysis of GOBP indicated that the highly secreted proteins in PC3 were significantly enriched in response to angiogenesis and platelet degranulation. The KEGG pathway enrichment revealed that lysosome-related secretory proteins were significantly enhanced in DU145. The domain annotation enrichment from InterPro results indicated that EGF-like domain was significantly concentrated in DU145. Previous studies had revealed that one of the well-known EGF-like domains is laminin, which affects cellular growth and mitogenic events $(22,23)$. In Figure 4, the STRING database was used to figure out the integrated protein-protein interactions (PPI) of differentially secreted proteins; results showed that proteins such as LAMC1, AGRN, HSPG2, and APP were highly inter-connected, dominant secretory proteins in DU145 whereas FN1 and INHBA proteins were the predominant secretory proteins of PC3. Based on these bioinformatics analysis, we focused on laminin $\mathrm{C}$ and laminin-binding in GOMF, to understand the specific characteristics of DU145 cells.

Prostate-cancer related mutant peptides in conditioned media. To identify the mutant peptides associated with prostate cancer, MS/MS spectra were searched using CMPD (downloaded from http://cgbc.cgu.edu.tw/cmpd/) that includes 24,189 prostate cancer-related mutant proteins (Figure 1C). Although possibilities of detecting mutant peptides are higher when a large number of databases are used, the number of false positives also tends to increase in such cases. For this reason, only the prostate cancer-related database was downloaded for use.

Initially, 42 peptides on 36 mutant proteins were identified, and verified manually following a process described in a previous study (24). Eventually, we identified six possible mutant peptides related to prostate cancer (Table I), of which, three were not quantified, and one had no significantly different secretion across the two prostate cancer cell lines. However, we confirmed that the two peptides, DTEEEDFHVDQATTVK from alpha-1-antitrypsin and SCCSCCPVDCAK from metallothionein-1E, were greatly increased in DU145 compared to those in PC3 cells. Unusually, BAG family molecular chaperone regulator 3 had no mutant sites. Nevertheless, we assumed that GQVAAAAAAQPPASHGPER is generated by trypsin cleavage, because cysteine $(C)$ in front of the sequence was mutated to arginine $(\mathrm{R})$.

\section{Discussion}

Prostate cancer (PCa) is the most common non-cutaneous malignancy in men. In particular, most patients with prostate cancer progress to metastasis in different parts $(2,3)$. The lack of biomarkers to replace PSA and dearth of understanding of the factors and mechanisms, involved in metastasis, pose the biggest challenges in this regard. To resolve this big issue, we designed MS-based secretory proteomics using metastatic PCa cell lines, DU145 and PC3. Among the various PCa cells, DU145 and PC3 cells lack PSA, $5 \alpha$-reductase, and androgen receptor (AR), hence are related to androgen-independent (AI) prostate cancers. Both cells are representatives of metastatic $\mathrm{PCa}$, but the metastatic tissues are different; DU145 and PC3 were originally isolated from brain and bone metastasis, respectively, in a patient with prostate cancer. PC3 is known to be a more aggressive prostate cancer cell than DU145 (25-27). Also, recent study showed that invasion of $\mathrm{PC} 3$ was enhanced by LNCaP co-culture, whereas DU-145 was inhibited (28). Hence, two different AI prostate cancer cells, DU145 and PC3, have unlike properties.

We investigated the potential metastatic and/or aggressive factors in secretory proteins from different prostate cancer cell lines by an MS-based proteomics approach. Particularly, TMT was used for quantitative proteomic analysis. TMT, an isobaric chemical labeling method, is a powerful and convenient tool to estimate the relative abundance of proteins. In particular, isobaric tagging strategy with MSbased proteins is suitable for low-abundance proteins, such as the ones secreted in CM, due to high signal from native peptides (29). We implemented quality check for the proteins, both from CM and from MS-based proteomics with TMT labeling, via western blot and scatter plot, respectively. Our results also showed that $\beta$-actin, but not $\alpha$-tubulin, exists in proteins from CM (Figure 1B). However, recent research has shown that $\beta$-actin may be secreted into the cell culture medium through an exosome-independent mechanism that is regulated by immune pathways (30). Pearson correlation coefficient between reporter ion intensities, examined to obtain the quantitative accuracy of MS-based proteomics, showed significance with an $\mathrm{R}$ value of 0.8 or higher (Figure 2A). Therefore, these results indicate that our proteomics results do have experimental significance.

We obtained 211 (37.6\%) differentially expressed secretory proteins (DEP), out of the total 561 of quantified proteins across DU145 and PC3 cells. In contrast to our proteomics results, which indicated that secretory proteins were considerably different between DU145 and PC3 cells, a previous study on cell-surface protein profiles of prostate cancer cell lines reported that expression of cell surface proteins (as seen by cluster of differentiation (CD)) is comparable in DU145 and PC because of similar biological 


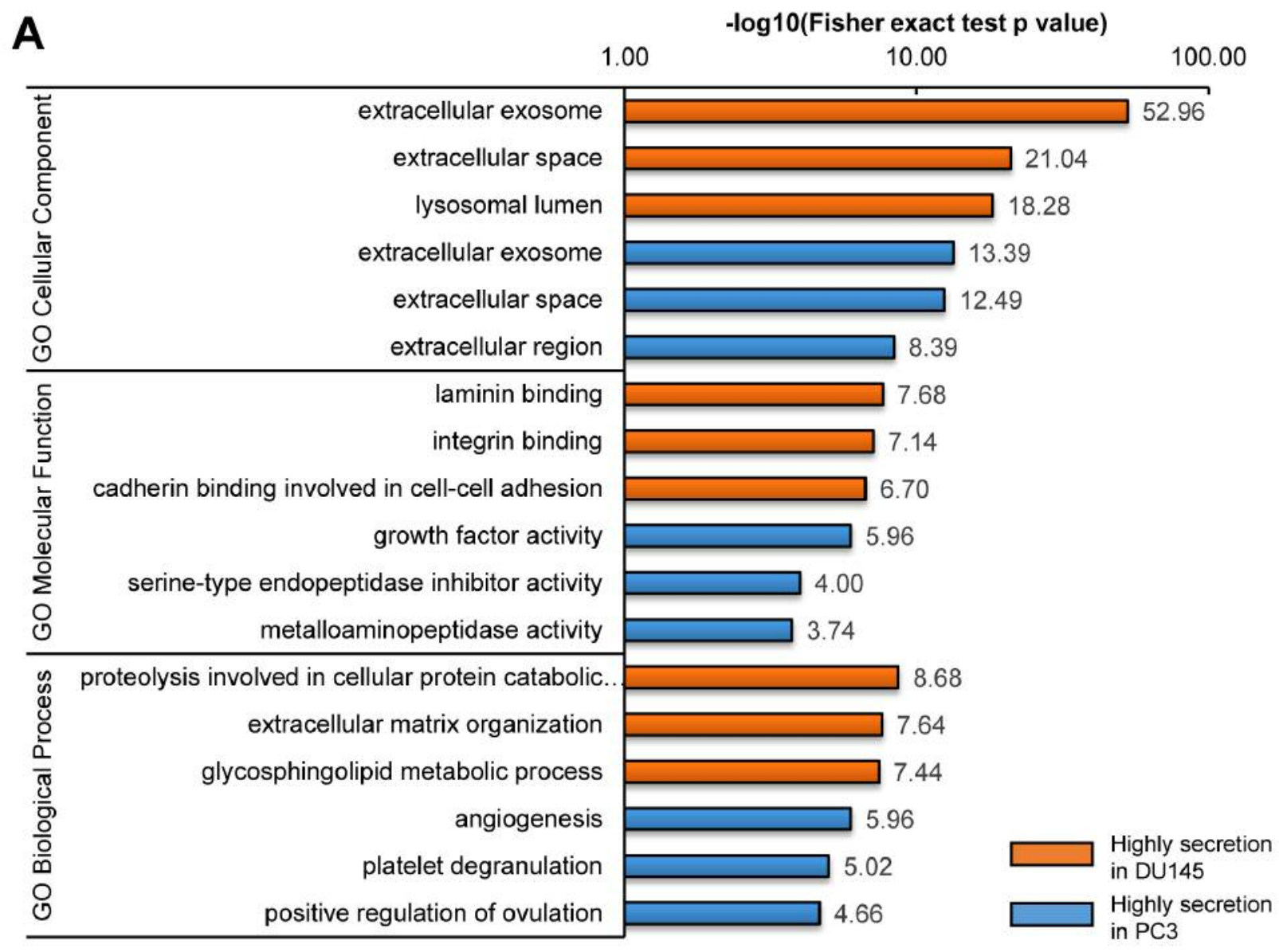

B

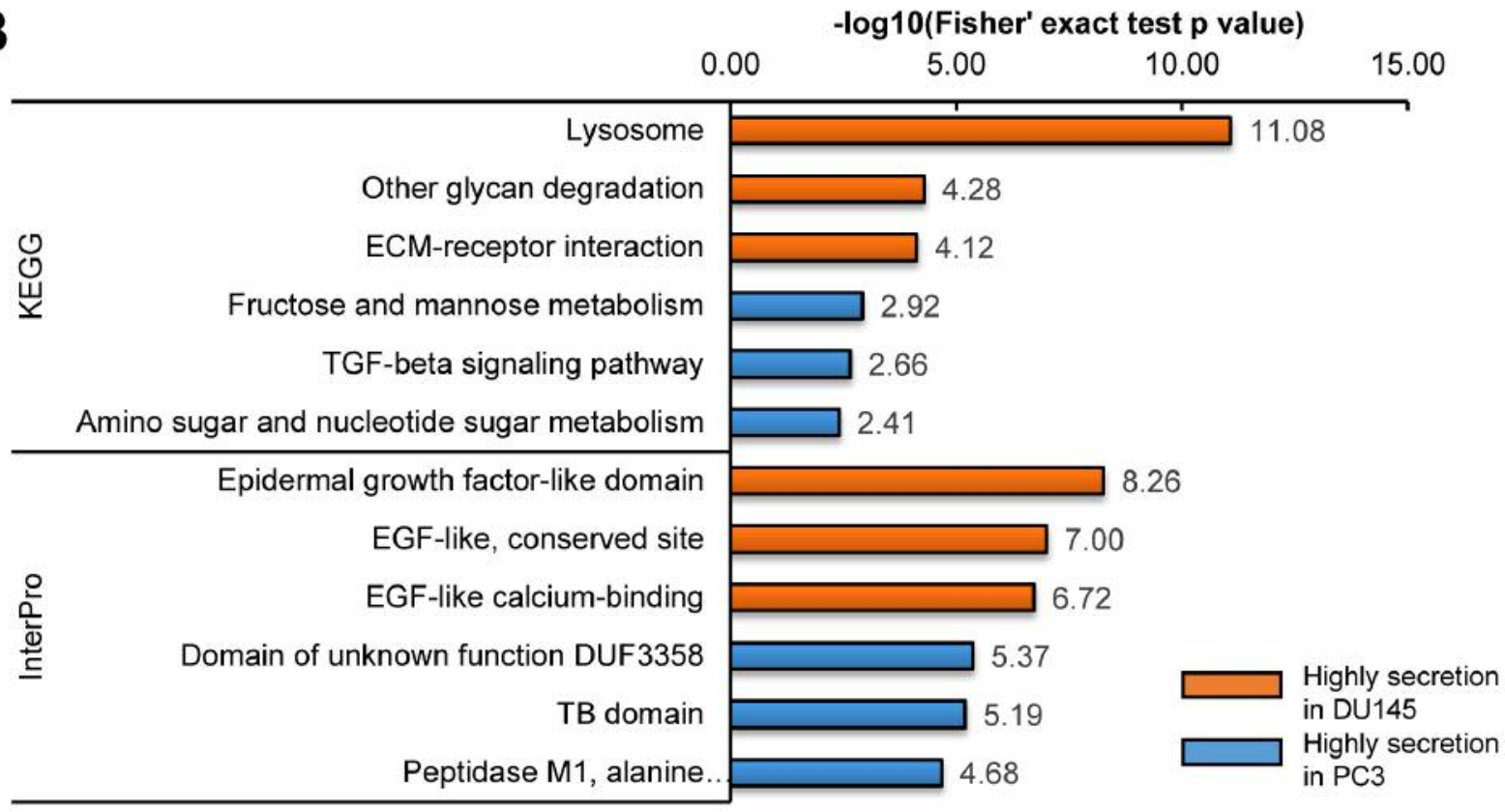

Figure 3. GO function (A), and protein domain and KEGG pathway (B) enrichment analysis of highly secreted proteins in DU145 and PC3 cells. 
A

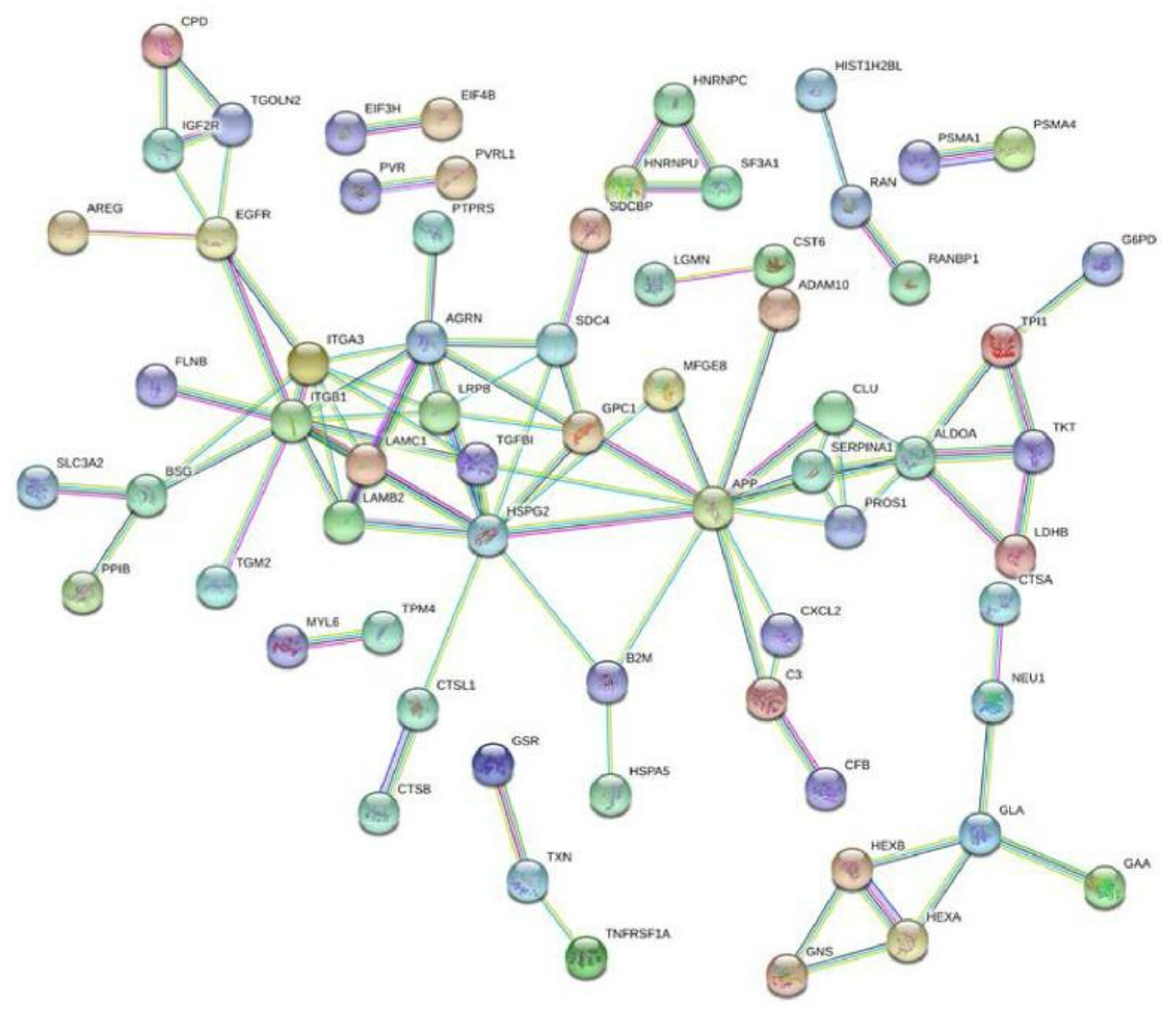

B

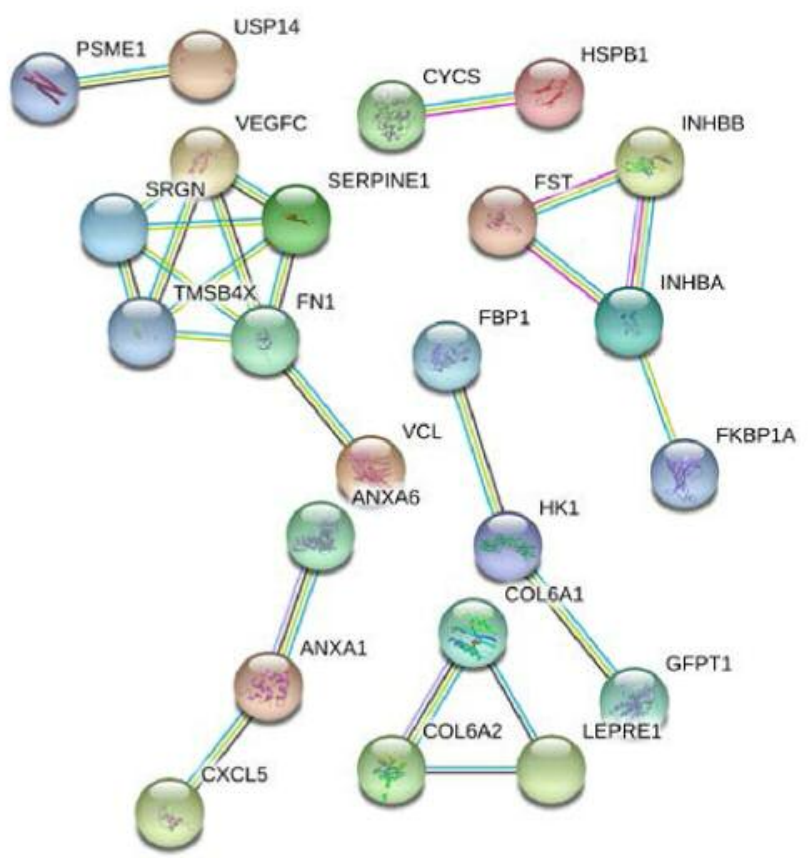

Figure 4. Protein-Protein Interaction network of highly secreted proteins in DU145 (A) and PC3 (B). 
Kwon et al: Comparative Secretome Profiling in Metastatic PCa Cells

Table I. List of identified and quantified mutant peptides in conditioned media of PC3 and DU145 cells.

\begin{tabular}{|c|c|c|c|c|c|c|c|c|}
\hline NCBInr ID & dbSNP ID & Uniprot ID & Gene name & Protein name & Mutation & Sequence & Score & $\begin{array}{c}\text { Log2 ratio } \\
\text { (DU145/ } \\
\text { PC3) }\end{array}$ \\
\hline NP_004272 & rs2234962 & O95817 & $B A G 3$ & $\begin{array}{l}\text { BAG family molecular } \\
\text { chaperone regulator } 3\end{array}$ & C151R & GQVAAAAAAQPPASHGPER & 40.968 & -0.358 \\
\hline NP_001121179 & rs6647 & P01009 & SERPINA1 & Alpha-1-antitrypsin & V237A & DTEEEDFHVDQ_ATTVK & 51.591 & 2.634 \\
\hline NP_783316 & rs72818417 & P04732 & $M T 1 E$ & Metallothionein-1E & G40D & SCCSCCPVDCAK & 79.492 & 3.516 \\
\hline NP_899199 & rs1049674 & P08243 & ASNS & Asparagine synthetase & V210E & DEPLHALYDNVEK & 45.28 & - \\
\hline NP_542380 & rs1130409 & P27695 & APEX1 & $\begin{array}{l}\text { DNA-(apurinic or } \\
\text { apyrimidinic site) } \\
\text { lyase }\end{array}$ & D148E & VSYGIGEEEHDQEGR & 49.988 & - \\
\hline NP_003237 & rs 2292305 & P07996 & THBS1 & Thrombospondin-1 & T523A & 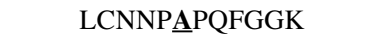 & 61.344 & - \\
\hline
\end{tabular}

Bold and underscore means mutated site.

behavior and gene expression (31). Among the secretory proteins, serpin B3 and LamC1 were used to verify the quantitative proteomics results using western blot; there seems to be a correlation between the results (Figure 2C). Serpin B3 appeared in both cell extract and CM of DU145 and PC3 cells. Serpin B3 is well-known in the positive regulation of cell migration and proliferation, hence implicating a role in invasiveness (32). Moreover, previous studies had indicated that high expression of serpin B3 could be utilized as a favorable, predictive, and prognostic biomarker in diverse forms of cancer, such as squamous cell carcinoma (SCC), breast cancer, and hepatocellular carcinoma (33-35). Our recent study also showed that Serpin B3 is over-secreted in PC3 cells, though not in normal prostate cell lines such as RWPE-1 (36).

Proteomics results were supplemented by bioinformatics analysis using DAVID and STRING for further understanding of the biological characteristics of DEP. Functional annotation enrichment, using DAVID, suggests that laminin and/or laminin-associated proteins have more potential biological functions in DU145 than in PC3 cells (Figure 3A). Furthermore, results of Protein-Protein interaction (PPI), via STRING, demonstrated that Laminin subunit gamma-1 (LAMC1) could be connected to the proteins highly secreted by DU145 cells, except for those known to be vastly expressed in the brain, like the Amyloid beta A4 protein (AAP) (Figure 4A). For this reason, we focused on LAMC1 which is specifically secreted from DU145, rather than PC3, but not expressed in cell extract.

LAMC1 acts as an oncogene located either in extracellular region or secreted. Indeed, invading tumor cells attach to laminin and increase their metastatic potential (37). Thus, LAMC1 was seen to significantly increase tumor cell invasion in hepatocellular carcinoma (38), and overexpressed in grade III meningioma compared to that in grade I (39). In a most recent study, $L A M C 1$ gene was up-regulated in uterine carcinomas, revealing clinically aggressive phenotypes (40). A previous study already revealed that silencing of LAMC1 by $m i R-29$ significantly inhibited cell migration and invasion in cancer cells. Conversely, up-regulated LamC1 in PC cells affects cell proliferation, migration, and invasion in $\mathrm{PCa}$ cell lines (41). Another report concerning LAMC1 at PCa indicated LAMC1 as direct target of $m i R-22$ (related to apoptosis and cell migration) (42). However, previous LAMC1 studies were all conducted in cell extracts, not on secreted LAMC1. Therefore, the present study confirmed that LAMC1 was secreted at a higher level in CM than in CE, more so in DU145 than in PC3. Hence, highly secreted LAMC1 might be suggested as a novel biomarker of predictive and prognostic metastases in PCa.

In this study, we used proteogenomics that integrates proteomics and genomics to discover a novel metastatic mutation associated with prostate cancer (Figure 1C). One of the major causes of cancer is the accumulated mutations in key proteins, which modulate cell growth, differentiation, and death (43). Mutations that change the protein sequence significantly affect protein stability and function. Indeed, proteins having PCa-associated mutations include BRCA1, BRCA2, and HOXB13, that may cause a higher risk of aggressive prostate cancer $(44,45)$. The HOXB13 protein, with G84E mutation, is associated with an increased risk of prostate cancer and other malignancies (46). Thus, the mutant proteins and/or sites enable us to look for new hallmarks of cancer (47). For this reason, we hypothesized that the secreted mutant proteins could be potent as new biomarkers with metastatic and/or distinguishable factors between PC3 and DU145 cells through a secretome analysis.

MS/MS results, re-analyzed using CMPD including largescale coding variants for cancer, have provided a collection of mutant protein sequences that correspond to large-scale 
genome data from NCI and TCGA. As a result, we identified six mutant secretory peptides in prostate cancer using MSbased proteomics combined with CMPD. The false-positive and non-mutant peptides were carefully verified and manually removed. Hence, only three out of six mutant peptides were quantified, of which two were found predominantly increased in DU145 (Table I). Researchers assume that due to gene aberrations, many protein sequence get altered, thereby increasing the proteasomal degradation of the incorrectly folded protein, subsequently, generating low abundance of mutant protein (48). Therefore, it follows that more aggressive PCa in bone metastatic PC 3 cell lines would have a lower level of mutant protein than in DU145. Unfortunately, the six identified mutant peptides, classified under single nucleotide polymorphism (SNP), contained missense mutation that is not reported to be implicated in the disease. Recently, millions of SNPs involved with diseases, but biological mechanisms and functions are still not understood $(49,50)$. Nevertheless, genome-wide association studies (GWAS) have been recently reported the 76 SNPs associated with prostate cancer (51). Other recent studies have shown that patient with CASP9 polymorphism (rs1052576) were related with increasing prostate cancer risk and the common SPOP mutant, F133V variant, increases invasion in prostate cancer cells $(52,53)$. Although the details regarding the increased preference of the six mutation sites in DU145 is not yet clear, the mutant peptides in $\mathrm{PCa}$ secretome are expected to provide an ideal opportunity for metastatic biomarker evaluation, using the evolving proteomics technologies such as selective (SRM) and Parallel reaction monitoring (PRM) $(54,55)$. Hence, lately some of papers were already indicated that Ras-mutant peptides as cancer-specific biomarkers identified and quantified in clinical specimens by MS-based proteomics approach as well as proteogeonimis revealed that TP53 mutation related to signal phosphorylation of MASTL and EEF2K in breast cancer $(56,57)$. Furthermore, mutant peptides can be used for tumor-associated antigens to apply to cancer peptide vaccines that utilize the clinical immunotherapies $(58,59)$.

In summary, we performed the quantitative proteomic analysis relevant to different metastatic sites associated with prostate cancer. In total, 598 proteins were identified, among which 561 were quantified based on the proteomic analysis. Among the quantified proteins, 128 were up-regulated and 83 were down-regulated in DU145 compared to PC3 cells. Based on our current results, LAMC1 was found to be highly over-expressed and specifically secreted in PCa cell lines; preferentially in DU145, indicating its potential as a biomarker to compare DU145 with PC3. The six newlyidentified mutations, besides being potent as specific markers for prostate cancer, are especially expected to be markers to distinguish the site of metastasis. In this regard, future studies should aim at exploring how LAMC1 is hypersecreted and distinguishes between DU145 and PC3prostate cancer cell lines. The identified mutant peptides should be confirmed as novel prostate cancer markers that can be identified from patient samples by SRM or PRM methods. In conclusion, we anticipate that the identified metastatic factors, LAMC1 and the six mutant peptides, could be used as biomarkers in the prognosis as well as new drug targets of metastatic prostate cancer.

\section{Conflicts of Interest}

The Authors declare that they have no competing interests.

\section{Acknowledgements}

This work was supported by the National Research Foundation of Korea (NRF) grant, funded by the Korean government (MSIP) (No.2015R1A2A2A01004286 and 2018R1D1A1A02043591).

\section{References}

1 Siegel RL, Miller KD and Jemal A: Cancer statistics, 2016. CA Cancer J Clin 66(1): 7-30, 2016.

2 Bienz M and Saad F: Management of bone metastases in prostate cancer: A review. Curr Opin Support Palliat Care 9(3): 261-267, 2015.

3 Jin JK, Dayyani F and Gallick GE: Steps in prostate cancer progression that lead to bone metastasis. Int J Cancer 128(11): 2545-2561, 2011.

4 Chiu KH, Chang YH, Wu YS, Lee SH and Liao PC: Quantitative secretome analysis reveals that col6a1 is a metastasis-associated protein using stacking gel-aided purification combined with itraq labeling. J Proteome Res 10(3): 1110-1125, 2011.

5 Hanash SM, Pitteri SJ and Faca VM: Mining the plasma proteome for cancer biomarkers. Nature 452(7187): 571-579, 2008.

6 Barderas R, Mendes M, Torres S, Bartolome RA, LopezLucendo M, Villar-Vazquez R, Pelaez-Garcia A, Fuente E, Bonilla $\mathrm{F}$ and Casal JI: In-depth characterization of the secretome of colorectal cancer metastatic cells identifies key proteins in cell adhesion, migration, and invasion. Mol Cell Proteomics 12(6): 1602-1620, 2013.

7 Lee E, Fertig EJ, Jin K, Sukumar S, Pandey NB and Popel AS: Breast cancer cells condition lymphatic endothelial cells within pre-metastatic niches to promote metastasis. Nat Commun 5: 4715, 2014.

8 Karagiannis GS, Pavlou MP and Diamandis EP: Cancer secretomics reveal pathophysiological pathways in cancer molecular oncology. Mol Oncol 4(6): 496-510, 2010.

9 Guo J, Yang J, Zhang X, Feng X, Zhang H, Chen L, Johnson H, Persson JL and Xiao K: A panel of biomarkers for diagnosis of prostate cancer using urine samples. Anticancer Res 38(3): 14711477, 2018.

10 Tanase CP, Codrici E, Popescu ID, Mihai S, Enciu AM, Necula LG, Preda A, Ismail G and Albulescu R: Prostate cancer proteomics: Current trends and future perspectives for biomarker discovery. Oncotarget 8(11): 18497-18512, 2017. 
11 Alfaro JA, Sinha A, Kislinger T and Boutros PC: Oncoproteogenomics: Cancer proteomics joins forces with genomics. Nat Methods 11(11): 1107-1113, 2014.

12 Sheynkman GM, Shortreed MR, Cesnik AJ and Smith LM: Proteogenomics: Integrating next-generation sequencing and mass spectrometry to characterize human proteomic variation. Annu Rev Anal Chem (Palo Alto Calif) 9(1): 521-545, 2016.

13 Allmer J: Algorithms for the de novo sequencing of peptides from tandem mass spectra. Expert Rev Proteomics 8(5): 645-657, 2011.

14 Yang X and Lazar IM: Xman: A homo sapiens mutated-peptide database for the ms analysis of cancerous cell states. J Proteome Res 13(12): 5486-5495, 2014.

15 Zhang M, Wang B, Xu J, Wang X, Xie L, Zhang B, Li Y and Li J: Canprovar 2.0: An updated database of human cancer proteome variation. J Proteome Res 16(2): 421-432, 2017.

16 Huang PJ, Lee CC, Tan BC, Yeh YM, Julie Chu L, Chen TW, Chang KP, Lee CY, Gan RC, Liu H and Tang P: CMPD: Cancer mutant proteome database. Nucleic Acids Res 43(Database issue): D849-855, 2015.

17 Cox J and Mann M: Maxquant enables high peptide identification rates, individualized p.P.B.-range mass accuracies and proteome-wide protein quantification. Nat Biotechnol 26(12): 1367-1372, 2008.

18 Uniprot: The universal protein knowledgebase. Nucleic Acids Res 45(D1): D158-D169, 2017.

19 Tyanova S, Temu T, Sinitcyn P, Carlson A, Hein MY, Geiger T, Mann M and Cox J: The perseus computational platform for comprehensive analysis of (prote)omics data. Nat Methods 13(9): 731-740, 2016.

20 Huang da W, Sherman BT and Lempicki RA: Systematic and integrative analysis of large gene lists using david bioinformatics resources. Nat Protoc 4(1): 44-57, 2009.

21 Szklarczyk D, Franceschini A, Wyder S, Forslund K, Heller D, Huerta-Cepas J, Simonovic M, Roth A, Santos A, Tsafou KP, Kuhn M, Bork P, Jensen LJ and von Mering C: String v10: Protein-protein interaction networks, integrated over the tree of life. Nucleic Acids Res 43(Database issue): D447-452, 2015.

22 Engel J: Egf-like domains in extracellular matrix proteins: Localized signals for growth and differentiation? FEBS Lett 251(1-2): 1-7, 1989.

23 Polna I and Aleksandrowicz J: Effect of adsorbents on igm and igg measles antibodies. Acta Virol 19(6): 449-456, 1975.

24 Chen Y, Kwon SW, Kim SC and Zhao Y: Integrated approach for manual evaluation of peptides identified by searching protein sequence databases with tandem mass spectra. J Proteome Res 4(3): 998-1005, 2005.

25 Stone KR, Mickey DD, Wunderli H, Mickey GH and Paulson DF: Isolation of a human prostate carcinoma cell line (du 145). Int J Cancer 21(3): 274-281, 1978.

26 Kaighn ME, Narayan KS, Ohnuki Y, Lechner JF and Jones LW: Establishment and characterization of a human prostatic carcinoma cell line (pc-3). Invest Urol 17(1): 16-23, 1979.

27 Russell PJ and Kingsley EA: Human prostate cancer cell lines Methods Mol Med 81: 21-39, 2003.

28 Takezawa Y, Izumi K, Machioka K, Iwamoto H, Naito R, Makino T, Kadomoto S, Natsagdorj A, Kadono Y, Keller ET, Zhang J and Mizokami A: Crosstalk between androgen-sensitive and androgen-insensitive prostate cancer cells. Anticancer Res 38(4): 2045-2055, 2018.
29 Rauniyar N and Yates JR, 3rd: Isobaric labeling-based relative quantification in shotgun proteomics. J Proteome Res 13(12): 5293-5309, 2014.

30 Sandiford SL, Dong Y, Pike A, Blumberg BJ, Bahia AC and Dimopoulos G: Cytoplasmic actin is an extracellular insect immune factor which is secreted upon immune challenge and mediates phagocytosis and direct killing of bacteria, and is a plasmodium antagonist. PLoS Pathog 11(2): e1004631, 2015.

31 Liu AY: Differential expression of cell surface molecules in prostate cancer cells. Cancer Res 60(13): 3429-3434, 2000.

32 Quarta S, Vidalino L, Turato C, Ruvoletto M, Calabrese F, Valente M, Cannito S, Fassina G, Parola M, Gatta A and Pontisso P: Serpinb3 induces epithelial-mesenchymal transition. J Pathol 221(3): 343-356, 2010.

33 Petty RD, Kerr KM, Murray GI, Nicolson MC, Rooney PH, Bissett D and Collie-Duguid ES: Tumor transcriptome reveals the predictive and prognostic impact of lysosomal protease inhibitors in non-small-cell lung cancer. J Clin Oncol 24(11): 1729-1744, 2006.

34 Collie-Duguid ES, Sweeney K, Stewart KN, Miller ID, Smyth E and Heys SD: Serpinb3, a new prognostic tool in breast cancer patients treated with neoadjuvant chemotherapy. Breast Cancer Res Treat 132(3): 807-818, 2012.

35 Turato C, Vitale A, Fasolato S, Ruvoletto M, Terrin L, Quarta S, Ramirez Morales R, Biasiolo A, Zanus G, Zali N, Tan PS, Hoshida Y, Gatta A, Cillo U and Pontisso P: Serpinb3 is associated with TGF-beta1 and cytoplasmic beta-catenin expression in hepatocellular carcinomas with poor prognosis. $\mathrm{Br}$ J Cancer 110(11): 2708-2715, 2014.

36 Sung E, Kwon OK, Lee JM and Lee S: Proteomics approach to identify novel metastatic bone markers from the secretome of PC-3 prostate cancer cells. Electrophoresis 38(20): 2638-2645, 2017.

37 Givant-Horwitz V, Davidson B and Reich R: Laminin-induced signaling in tumor cells. Cancer Lett 223(1): 1-10, 2005.

38 Zhang Y, Xi S, Chen J, Zhou D, Gao H, Zhou Z, Xu L and Chen $\mathrm{M}$ : Overexpression of LAMC1 predicts poor prognosis and enhances tumor cell invasion and migration in hepatocellular carcinoma. J Cancer 8(15): 2992-3000, 2017.

$39 \mathrm{Ke} \mathrm{HL}, \mathrm{Ke} \mathrm{RH}, \mathrm{Li} \mathrm{B}$, Wang XH, Wang YN and Wang XQ: Association between laminin gammal expression and meningioma grade, recurrence, and progression-free survival. Acta Neurochir (Wien) 155(1): 165-171, 2013.

40 Kashima H, Wu RC, Wang Y, Sinno AK, Miyamoto T, Shiozawa T, Wang TL, Fader AN and Shih Ie M: Laminin c1 expression by uterine carcinoma cells is associated with tumor progression. Gynecol Oncol 139(2): 338-344, 2015.

41 Nishikawa R, Goto Y, Kojima S, Enokida H, Chiyomaru T, Kinoshita T, Sakamoto S, Fuse M, Nakagawa M, Naya Y, Ichikawa $\mathrm{T}$ and Seki N: Tumor-suppressive microRNA-29s inhibit cancer cell migration and invasion via targeting lamc1 in prostate cancer. Int J Oncol 45(1): 401-410, 2014.

42 Pasqualini L, Bu H, Puhr M, Narisu N, Rainer J, Schlick B, Schafer G, Angelova M, Trajanoski Z, Borno ST, Schweiger MR, Fuchsberger C and Klocker H: Mir-22 and mir-29a are members of the androgen receptor cistrome modulating LAMC1 and MCL-1 in prostate cancer. Mol Endocrinol 29(7): 10371054, 2015

43 Loeb KR and Loeb LA: Significance of multiple mutations in cancer. Carcinogenesis 21(3): 379-385, 2000. 
44 Ostrander EA and Udler MS: The role of the BRCA2 gene in susceptibility to prostate cancer revisited. Cancer Epidemiol Biomarkers Prev 17(8): 1843-1848, 2008.

45 Taylor RA, Fraser M, Livingstone J, Espiritu SM, Thorne H, Huang V, Lo W, Shiah YJ, Yamaguchi TN, Sliwinski A, Horsburgh S, Meng A, Heisler LE, Yu N, Yousif F, Papargiris M, Lawrence MG, Timms L, Murphy DG, Frydenberg M, Hopkins JF, Bolton D, Clouston D, McPherson JD, van der Kwast T, Boutros PC, Risbridger GP and Bristow RG: Germline BRCA2 mutations drive prostate cancers with distinct evolutionary trajectories. Nat Commun 8: 13671, 2017.

46 Beebe-Dimmer JL, Hathcock M, Yee C, Okoth LA, Ewing CM, Isaacs WB, Cooney KA and Thibodeau SN: The HOXB13 G84e mutation is associated with an increased risk for prostate cancer and other malignancies. Cancer Epidemiol Biomarkers Prev 24(9): 1366-1372, 2015.

47 Hanahan D and Weinberg RA: Hallmarks of cancer: The next generation. Cell 144(5): 646-674, 2011.

48 Freed-Pastor WA and Prives C: Mutant p53: One name, many proteins. Genes Dev 26(12): 1268-1286, 2012.

49 Bush WS and Moore JH: Chapter 11: Genome-wide association studies. PLoS Comput Biol 8(12): e1002822, 2012.

50 Ruggles KV, Krug K, Wang X, Clauser KR, Wang J, Payne SH, Fenyo D, Zhang B and Mani DR: Methods, tools and current perspectives in proteogenomics. Mol Cell Proteomics 16(6): 959-981, 2017.

51 Eeles R, Goh C, Castro E, Bancroft E, Guy M, Al Olama AA, Easton D and Kote-Jarai Z: The genetic epidemiology of prostate cancer and its clinical implications. Nat Rev Urol 11(1): 18-31, 2014.

52 Yilmaz SG, Yencilek F, Yildirim A, Yencilek E and Isbir T: Effects of caspase 9 gene polymorphism in patients with prostate cancer. In Vivo 31(2): 205-208, 2017.

53 Barbieri CE, Baca SC, Lawrence MS, Demichelis F, Blattner M, Theurillat JP, White TA, Stojanov P, Van Allen E, Stransky N, Nickerson E, Chae SS, Boysen G, Auclair D, Onofrio RC, Park K, Kitabayashi N, MacDonald TY, Sheikh K, Vuong T, Guiducci C, Cibulskis K, Sivachenko A, Carter SL, Saksena G, Voet D, Hussain WM, Ramos AH, Winckler W, Redman MC, Ardlie K, Tewari AK, Mosquera JM, Rupp N, Wild PJ, Moch H, Morrissey C, Nelson PS, Kantoff PW, Gabriel SB, Golub TR, Meyerson M, Lander ES, Getz G, Rubin MA and Garraway LA: Exome sequencing identifies recurrent SPOP, FOXA1 and MED12 mutations in prostate cancer. Nat Genet 44(6): 685-689, 2012.
54 Picotti P, Rinner O, Stallmach R, Dautel F, Farrah T, Domon B, Wenschuh $\mathrm{H}$ and Aebersold R: High-throughput generation of selected reaction-monitoring assays for proteins and proteomes. Nat Methods 7(1): 43-46, 2010.

55 Mathivanan S, Ji H, Tauro BJ, Chen YS and Simpson RJ: Identifying mutated proteins secreted by colon cancer cell lines using mass spectrometry. J Proteomics 76: Spec No. 141-149, 2012.

56 Wang Q, Chaerkady R, Wu J, Hwang HJ, Papadopoulos N, Kopelovich L, Maitra A, Matthaei H, Eshleman JR, Hruban RH, Kinzler KW, Pandey A and Vogelstein B: Mutant proteins as cancer-specific biomarkers. Proc Natl Acad Sci USA 108(6): 2444-2449, 2011.

57 Mertins P, Mani DR, Ruggles KV, Gillette MA, Clauser KR, Wang P, Wang X, Qiao JW, Cao S, Petralia F, Kawaler E, Mundt F, Krug K, Tu Z, Lei JT, Gatza ML, Wilkerson M, Perou CM, Yellapantula V, Huang KL, Lin C, McLellan MD, Yan P, Davies SR, Townsend RR, Skates SJ, Wang J, Zhang B, Kinsinger CR, Mesri M, Rodriguez H, Ding L, Paulovich AG, Fenyo D, Ellis MJ and Carr SA: Proteogenomics connects somatic mutations to signalling in breast cancer. Nature 534(7605): 55-62, 2016.

58 Schumacher TN and Schreiber RD: Neoantigens in cancer immunotherapy. Science 348(6230): 69-74, 2015.

59 Carreno BM, Magrini V, Becker-Hapak M, Kaabinejadian S, Hundal J, Petti AA, Ly A, Lie WR, Hildebrand WH, Mardis ER and Linette GP: Cancer immunotherapy. A dendritic cell vaccine increases the breadth and diversity of melanoma neoantigenspecific T cells. Science 348(6236): 803-808, 2015.
Received May 4, 2018

Revised June 4, 2018

Accepted June 6, 2018 